\title{
Dominant TOM1 mutation associated with combined immunodeficiency and autoimmune disease
}

Salla Keskitalo ${ }^{1}$, Emma M. Haapaniemi ${ }^{2,3,4}$, Virpi Glumoff ${ }^{5}$, Xiaonan Liu (D) ${ }^{1}$, Ville Lehtinen ${ }^{6}$, Christopher Fogarty ${ }^{7,8,9}$, Hanna Rajala ${ }^{10,11}$, Samuel C. Chiang ${ }^{12}$, Satu Mustjoki ${ }^{10,11}$, Panu Kovanen ${ }^{13}$, Jouko Lohi ${ }^{13}$, Yenan T. Bryceson ${ }^{3}$, Mikko Seppänen (D) ${ }^{14}$, Juha Kere ${ }^{7,15,16,17}$, Kaarina Heiskanen ${ }^{18}$ and Markku Varjosalo ${ }^{1}$

Mutations in several proteins functioning as endolysosomal components cause monogenic autoimmune diseases, of which pathogenesis is linked to increased endoplasmic reticulum stress, inefficient autophagy, and defective recycling of immune receptors. We report here a heterozygous TOM1 p.G307D missense mutation, detected by whole-exome sequencing, in two related patients presenting with early-onset autoimmunity, antibody deficiency, and features of combined immunodeficiency. The index patient suffered from recurrent respiratory tract infections and oligoarthritis since early teens, and later developed persistent lowcopy EBV-viremia, as well as an antibody deficiency. Her infant son developed hypogammaglobulinemia, autoimmune enteropathy, interstitial lung disease, profound growth failure, and treatment-resistant psoriasis vulgaris. Consistent with previous knowledge on TOM1 protein function, we detected impaired autophagy and enhanced susceptibility to apoptosis in patient-derived cells. In addition, we noted diminished STAT and ERK1/2 signaling in patient fibroblasts, as well as poor IFN- $\gamma$ and IL-17 secretion in T cells. The mutant TOM1 failed to interact with TOLLIP, a protein required for IL-1 recycling, PAMP signaling and autophagosome maturation, further strengthening the link between the candidate mutation and patient pathophysiology. In sum, we report here an identification of a novel gene, TOM1, associating with early-onset autoimmunity, antibody deficiency, and features of combined immunodeficiency. Other patient cases from unrelated families are needed to firmly establish a causal relationship between the genotype and the phenotype.

npj Genomic Medicine (2019)4:14; https://doi.org/10.1038/s41525-019-0088-5

\section{INTRODUCTION}

Primary immunodeficiencies that present with prominent autoimmunity provide insights into the molecular mechanisms of tolerance breakdown. The common defective genes in these conditions encode proteins that affect T-cell development and function. Examples include loss of FOXP3 that governs the regulatory T-cell development, and gain or loss of the STAT proteins that polarize $T$ cells to different helper subsets. ${ }^{1-8}$ In addition, defects in major immunological signaling molecules and proteins that govern the actin cytoskeleton formation can lead to T-cell driven autoimmunity. ${ }^{2,7}$

Several proteins functioning in the endolysosomal system can also cause monogenic autoimmune diseases when defective ${ }^{9-11}$ (online Supplementary Information Table S1). The pathogenesis is linked to increased endoplasmic reticulum stress, inefficient autophagy, and impaired recycling of immune receptors. Herein, we report a previously undescribed, dominantly inherited immune disease in two related patients that carry a missense mutation in TOM1 (Target of Myb protein 1). TOM1 is an adaptor protein needed for the maturation of autophagosomes and their fusion with lysosomes. ${ }^{12}$ TOM1 also inhibits Toll-like receptor (TLR) signaling and participates in immune receptor recycling. ${ }^{13,14}$ Clinically, the index patient presents with a relatively mild disease, whereas in the child the condition is aggressive and fatal. This phenotypic heterogeneity is common in monogenic immune diseases and points to additional genetic modifiers in disease presentation.

\section{RESULTS}

We evaluated a mother-son pair presenting with childhood-onset autoimmune disease and combined immunodeficiency (Table 1, and detailed case descriptions in Supplementary Information). The index case (Patient 1, II.2) suffered from recurrent respiratory tract

\footnotetext{
${ }^{1}$ Institute of Biotechnology, University of Helsinki, Helsinki, Finland; ${ }^{2}$ Centre for Molecular Medicine Norway, University of Oslo, Oslo, Norway; ${ }^{3}$ Center for Hematology and Regenerative Medicine, Karolinska Institutet, Stockholm, Sweden; ${ }^{4}$ Biomedicum Stem Cell Center, University of Helsinki, Helsinki, Finland; ${ }^{5}$ Research Unit of Biomedicine, Medical Microbiology and Immunology, University of Oulu, Oulu, Finland; ${ }^{6}$ Päijät-Häme Central Hospital, Lahti, Finland; ${ }^{7}$ Folkhälsan Institute of Genetics, Helsinki, Finland; ${ }^{8}$ Abdominal Center Nephrology, University of Helsinki and Helsinki University Hospital, Helsinki, Finland; ${ }^{9}$ Research Programs Unit, Diabetes, and Obesity, University of Helsinki, Helsinki, Finland; ${ }^{10}$ Hematology Research Unit Helsinki, Helsinki University Hospital Comprehensive Cancer Center, Helsinki, Finland; ${ }^{11}$ Department of Clinical Chemistry, University of Helsinki, Helsinki, Finland; ${ }^{12}$ Center for Hematology and Regenerative Medicine, Karolinska Institutet, Huddinge, Sweden; ${ }^{13}$ Department of Pathology, University of Helsinki and Helsinki University Hospital, Helsinki, Finland; ${ }^{14}$ Rare Disease Center, Hospital for Children and Adolescents and Adult Immunodeficiency Unit, Inflammation Center, University of Helsinki and Helsinki University Hospital, Helsinki, Finland; ${ }^{15}$ Department of Biosciences and Nutrition, Karolinska Institutet, Stockholm, Sweden; ${ }^{16}$ Research Programs Unit, Molecular Neurology, University of Helsinki, Helsinki, Finland; ${ }^{17}$ School of Basic and Medical Biosciences, King's College London, Guy's Hospital, London, UK and ${ }^{18}$ Hospital for Children and Adolescents, University of Helsinki and Helsinki University Hospital, Helsinki, Finland
}

Correspondence: Emma M. Haapaniemi (emma.haapaniemi@ki.se) or Markku Varjosalo (markku.varjosalo@helsinki.fi)

These authors contributed equally: Salla Keskitalo, Emma M. Haapaniemi

Received: 7 November 2018 Accepted: 4 June 2019

Published online: 27 June 2019 
Table 1. Clinical and immunological features of the study participants

\begin{tabular}{|c|c|c|}
\hline Sex & Female & Male \\
\hline Current age & 32 & 9 \\
\hline \multicolumn{3}{|l|}{ Infection susceptibility } \\
\hline & Respiratory tract infections & Respiratory tract infections \\
\hline & Atopic eczema & Psoriasis \\
\hline & Seronegative polyarthritis & Lymphocytic interstitial pneumonitis \\
\hline & Chronic diarrhea & Autoimmune enteropathy \\
\hline \multicolumn{3}{|l|}{ Laboratory features $^{a}$} \\
\hline Lymphocytes (1300-3600) & $660 \downarrow$ & 1670 \\
\hline T cells $(C D 3+$ CD8 +$)(200-1200)$ & $170 \downarrow$ & 300 \\
\hline $\mathrm{T}_{\text {reg }}(\mathrm{FOXP3}+\mathrm{CD} 25+)(2.8 \%-6.4 \%)$ & 5.8 & 5.5 \\
\hline$T_{\text {reg }}$ suppressive capacity & Normal & Reduced \\
\hline NK cells $(C D 3-C D 16+56+)(90-600)$ & $40 \downarrow$ & $20 \downarrow$ \\
\hline Plasmacytoid dendritic cells (lin-HLA-DR + CD123 + CD11c-) & $0.04 \downarrow$ & $0.04 \downarrow$ \\
\hline Monocytoid dendritic cells (lin-HLA-DR + CD13-CD11c +) & $0.04 \downarrow$ & $0.04 \downarrow$ \\
\hline \multicolumn{3}{|l|}{ Immunoglobulins } \\
\hline $\lg G(6.8-15.0 \mathrm{~g} / \mathrm{l})$ & $0.9 \downarrow$ & 7.5 (substitution) \\
\hline $\lg \mathrm{A}(0.52-4.02 \mathrm{~g} / \mathrm{l})$ & $0 \downarrow$ & $<0.1 \downarrow$ \\
\hline $\lg M(0.47-2.84 \mathrm{~g} / \mathrm{l})$ & $0.07 \downarrow$ & $0.1 \downarrow$ \\
\hline $\lg E(0-110$ IU/I) & $<4$ & $5 \mathrm{kU} / \mathrm{l}$ \\
\hline
\end{tabular}

infections and joint pain since her early teens, and was diagnosed with seronegative oligoarthritis and hypogammaglobulinemia at age 16 (plasma lgG of $3,0 \mathrm{~g} / \mathrm{l}, \lg A \quad 0,15 \mathrm{~g} / \mathrm{l}$, and $\operatorname{lgM} 0,17 \mathrm{~g} / \mathrm{l}$ ). Intravenous immunoglobulin replacement was initiated but soon discontinued due to adverse effects. In her early $30 \mathrm{~s}$, she developed chronic diarrhea with normal endoscopic findings and was noted to have persistent low-copy EBV viremia, between 200-800 viral copies/ml. She is currently receiving peroral prednisolone, methotrexate, and subcutaneous immunoglobulin replacement.

Her son (Patient 2, III.1) was evaluated for failure to thrive at age 6 months and was subsequently diagnosed with autoimmune enteropathy and lung disease that later evolved to lymphocytic interstitial pneumonitis (LIP). At age 3, he developed eczema that, by age 6 , had become generalized and treatment resistant, resembling psoriasis vulgaris (Supplementary Fig. S1). He displayed profound growth failure (-4.5 SD) and hypogammaglobulinemia since age 12 months. During the course of the study, he received peroral tacrolimus, methotrexate and subcutaneous immunoglobulin replacement, and recently underwent allogeneic stem cell transplant, with a temporary resolution of autoimmune symptoms. Within 6 months, however, he rejected the allograft and the disease returned. He died one year after the transplant for progressive pulmonary fibrosis.

Detailed immunological workup is presented in online Supplementary Information Tables S2 and S3. Both patients had low numbers of dendritic cells, NK cells and switched memory B cells; additionally, the regulatory T-cell function was impaired in the severely affected son (Table 1 and Tables S2 and S3). The T-cell compartment showed increased naive as well as decreased T-cell effector memory (TEM) and effector memory RA (TEMRA) subsets, suggesting impaired T-cell maturation. Both patients had poor IFN- $\gamma$ and IL-17 secretion upon stimulation, even though enhanced Th17 response is considered a hallmark of T-cellmediated autoimmune disease. ${ }^{6}$

As the clinical presentation pointed to dominantly inherited immunodeficiency, we performed trio-whole-exome sequencing of the mother, the only child and his healthy father. We filtered the data for dominant coding sequence variants shared by the affected individuals and not present in the public and in-house control datasets. This resulted in 13 variants, of which three were damaging to the protein structure and affected a conserved residue (online Supplementary Information Table S4). A variant in Target of Myb protein 1 -gene (TOM1; chr22: 35728994 G > A, p. G307D, Fig. 1a) was considered the most likely candidate, as it was absent from control datasets and deleteriously affected a conserved residue (SIFT prediction "deleterious", PolyPhen prediction "probably damaging"). TOM1 is highly expressed in immune cells and participates in autophagy, ubiquitination, and receptor recycling; pathways, which are implicated in several monogenic autoimmune conditions (refs ${ }^{10,11}$ and Table S1).

The expression of WT and mutant TOM1 were identical (Fig. 1b and Supplementary Fig. S1B); therefore, we hypothesized that the mutation affects cellular protein-protein interactions of TOM1, as is the case in many pathogenic missense variants that do not lead to absent protein expression. ${ }^{15}$ Therefore, we used Flp-In T-REx 


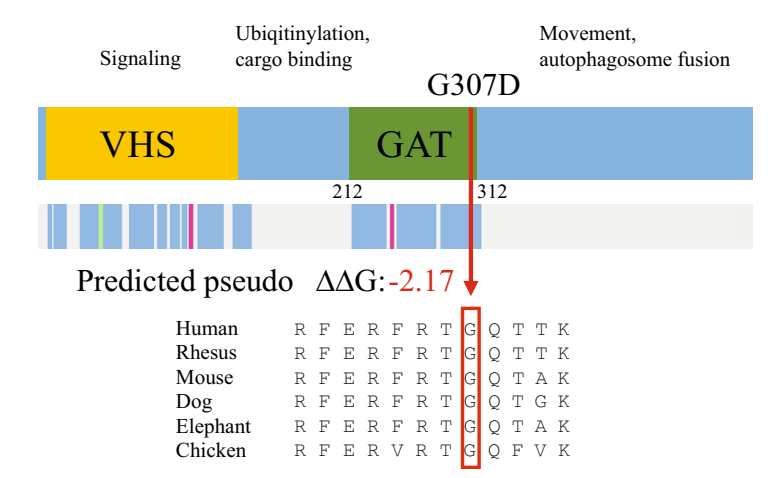

b

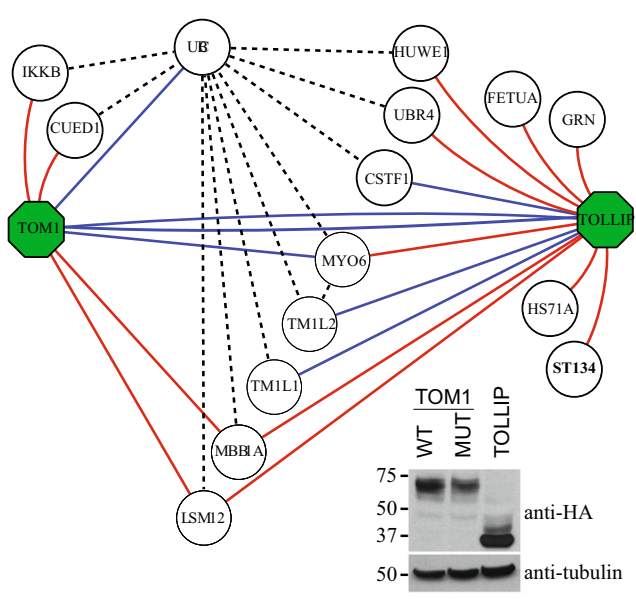

d

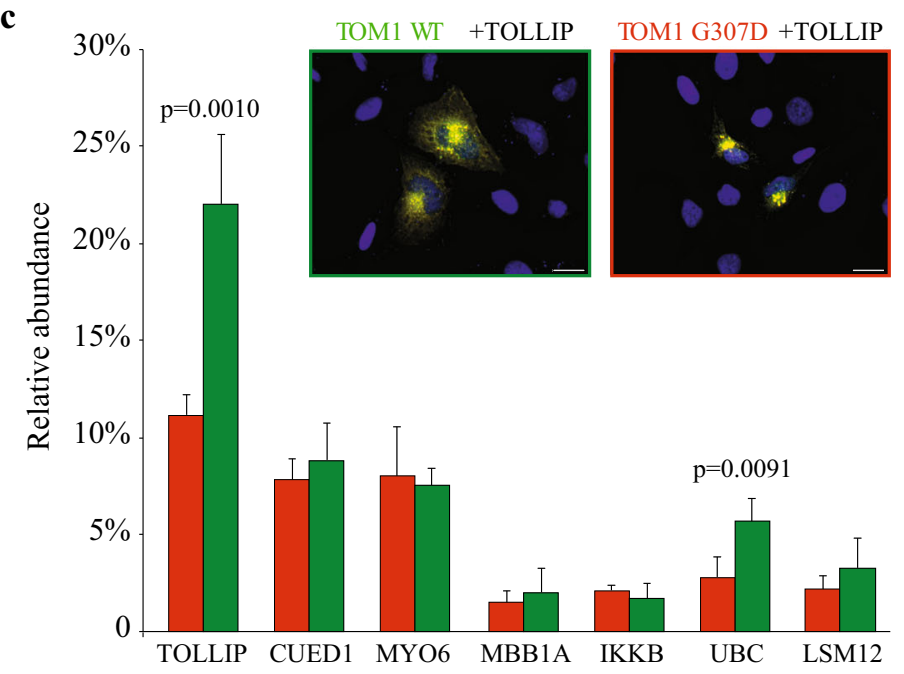

$\mathbf{e}$

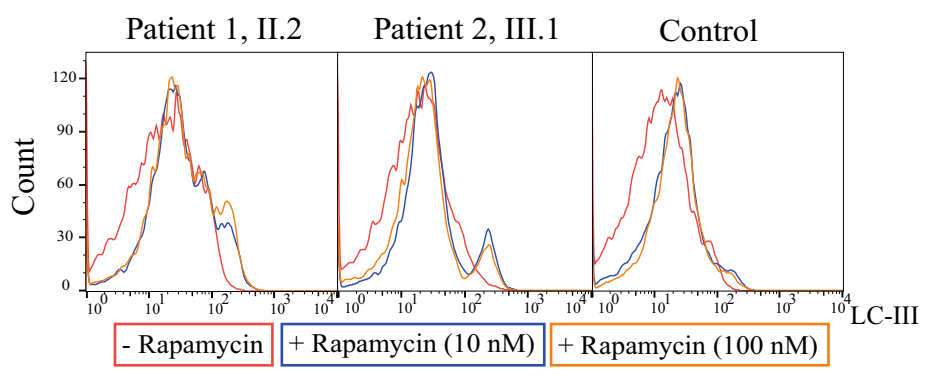

f

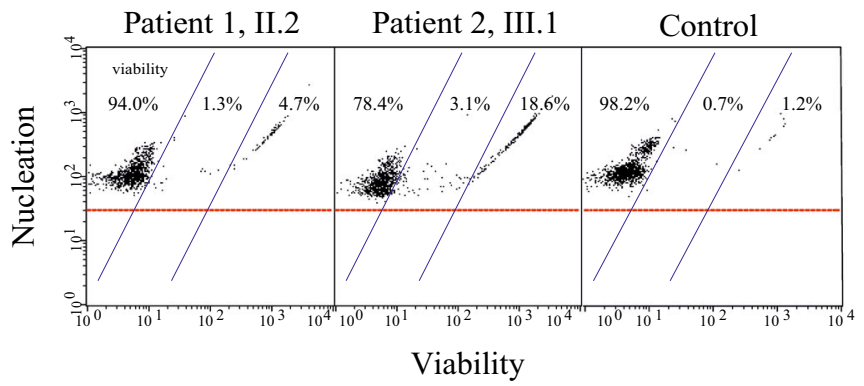

MFI

— Patient 1, II.2 (mother) 10.1

- Patient 2, III.1 (son) 8.7

- Healthy control $\quad 19.8$

- - unstained control 7.4

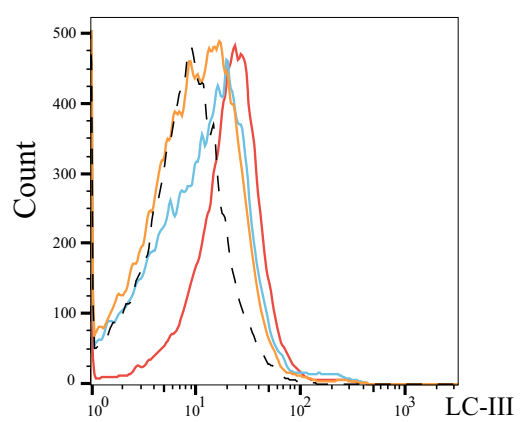

g
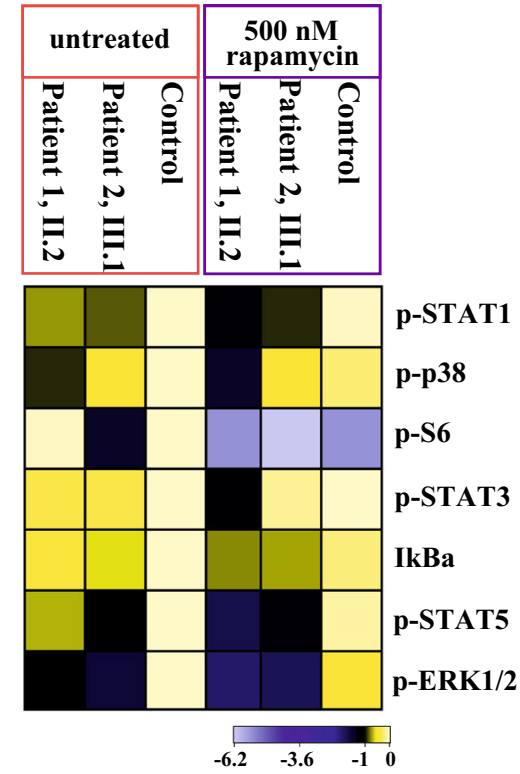

293 cell lines that ectopically expressed mutant or wild type (WT) streptavidin-tagged TOM1 and analyzed the interactions by affinity-purification mass spectrometry (AP-MS). The AP-MS analysis confirmed the known and identified several novel interactions (Fig. 1b). In the quantitative interactome analysis the mutant TOM1 had statistically significant decreased interactions with ubiquitin C (2.8\% mutant, 5.7\% WT) and TOLLIP $(11.2 \%$ mutant, $22.0 \%$ WT) (Fig. 1c). TOLLIP is an endosomal protein that negatively regulates TLR (toll-like receptor) and innate immune signaling, and is associated with various autoimmune 
Fig. 1 a Schematic representation of TOM1 and the observed mutation in the GAT (GGA and Tom1) domain. Mutated G307 residue and its evolutionary conservation is shown together with predicted reduced stability (predicted pseudo $\Delta \Delta G$ ). $\mathbf{b}$ The AP-MS analysis of TOM1 wild type (WT), G307D mutant and TOLLIP WT confirmed reciprocally the known interactions of TOM1 and TOLLIP (blue edges) as well as identified several novel (red) interactions. The black dashed line indicates known prey-to-prey interactions. Inset: expression of all the constructs was confirmed with western blotting using anti-HA antibody. Tubulin was used as a loading control. Blots derive from the same experiment and they were processed in parallel. c The quantitative interactome analysis of TOM1 WT (green) and G307D mutant (red) showed significantly decreased binding of the G307D with polyubiquitin-C (UBC) and TOLLIP compared to the WT. Error bars indicate standard deviation. The $p$ values were calculated using Student's $t$-test. Inset: TOLLIP efficiently recruits TOM1 WT (left) to early endosomes while recruitment of G307D (right) is hampered. HeLa cells were co-transfected with Myc-tagged WT or G307D TOM1 (green and red rectangle, respectively) and HAtagged TOLLIP. Merged images are shown, where scale bar represents $20 \mu \mathrm{m}$. d Diminished LC3 staining of patient PBMCs indicates decreased autophagy. Son (III.1) is indicated with orange, mother (II.2) with blue, and control with red solid line. Interestingly, 50\% of son's lymphocytes overlapped with the unstained control population (black dashed line). MFI, mean fluorescence intensity. e Rapamycin fails to induce autophagy in PBMCs. Line colors indicate rapamycin concentrations. f PBMCs show elevated numbers of apoptotic and dead cells in both patients. g Intracellular signaling network responses in patient skin fibroblasts reveals alterations of key pathway components phosphorylation levels at basal state and after rapamycin treatment. Heat map shows the calculated Log 2 ratios of medians of panel/ channel values relative to untreated control. Analyzed phosphoepitopes were STAT1 (anti-pTyr701), p38 (anti-pThr180/pTyr192), S6 (antipSer235/pSer236), STAT3 (anti-pTyr705), STAT5 (anti-pTyr694), and ERK1/2 (anti-pThr202/pTyr204)

conditions. $^{16}$ Normally upon transient co-expression TOLLIP recruits TOM1 to endosomes, ${ }^{13}$ however this function was impaired with the mutant TOM1 (Fig. 1c), although WT and mutant TOM1 showed similar cytosolic localization (Supplementary Fig. S2). These data suggest that the mutation disrupts the TOM1-TOLLIP complex formation, which then hampers the packaging of ubiqitinylated proteins to endosomes.

To further understand how TOM1-TOLLIP complex functions in endosomal cargo delivery, we performed BiolD experiments with stable Flp-In T-REx 293 cells lines. The BiolD method involves baitfused modified biotin ligase (BirA) biotinylating close-proximity proteins allowing identification of transient and proximal interactions. $^{17}$ In this analysis TOM1 and TOLLIP localized to endo/ exosomal vesicles and had multiple common interaction partners. As defects in the vesicular trafficking protein LRBA cause monogenic autoimmune disease similar to our patients, ${ }^{18}$ we even mapped the interactions of this protein. LRBA interacted with both TOM1 and TOLLIP but had molecular context mainly in the Golgi/trans-Golgi transport. This suggests that while TOM1 localizes to vesicles, LRBA functions mainly in the Golgiendosomal axis, and the molecular pathogenesis of TOM1 and LRBA deficiencies are distinct from each other (Supplementary Fig. S2).

Ubiqitinylated proteins are degraded by the endolysosomal system. The process is tightly connected to autophagy, in which the cell digests its own organelles to create energy and nutrients. To evaluate whether autophagy was disturbed in patient lymphocytes, we analyzed the expression of LC3 - which indicates the presence of autophagosomes-after serum starvation (Fig. 1d). Lymphocytes from both patients showed decreased LC3 staining, indicating a low autophagosome count compared to a healthy control. Rapamycin and other rapalogs are potent inducers of autophagy, and in an attempt to control autoimmunity we treated patient 2 with everolimus. Contrary to our expectations, this led to flares of eczema and respiratory distress, symptoms that quickly stabilized once the drug was stopped. In vitro, rapamycin treatment on patient lymphocytes failed to increase the number of autophagosomes (Fig. 1e). These observations point to impaired autophagy, most probably due to defects in autophagosome formation.

As impaired autophagy often leads to increased apoptosis, ${ }^{19}$ we also evaluated the proportion of apoptotic and dead PBMCs in the patients, which were increased compared to healthy control (Fig. 1f). Similar phenotypes of increased apoptosis and defective autophagy have been reported in common autoimmune conditions such as systemic lupus erythematosus and in the monogenic autoimmune disease caused by LRBA-deficiency. ${ }^{18,20}$ Impaired autophagy is also linked to increased endoplasmic reticulum stress; ${ }^{21}$ however, we did not detect evidence of abnormal ER stress response when staining inflamed gut and respiratory mucosa paraffin sections with BiP antibody.

Besides autophagy, TOM1 participates in receptor recycling. ${ }^{12,13}$ We therefore examined the patient lymphocytes for their ability to express CTLA4, IL- 1 and IL-6 receptors, as increased IL- 6 signaling and impaired CTLA-4 expression give monogenic autoimmune phenotypes similar to our patients. ${ }^{6,22}$ All tests were normal (Supplementary Fig. S2). To further understand the effect of the mutation on major immune signaling pathways, we measured activating phosphorylation of components of Jak-STAT, NF-KB, and Ras-MAPK signaling pathways in patient skin fibroblasts (Fig. 1g). Interestingly, ERK1/2 phosphorylation was significantly downregulated in both patients (log2 fold change $>1$, Fig. 1g) indicating dysfunctional MAPK signaling. Both patients also showed impaired STAT1 and STAT5 tyrosine phosphorylation that further diminished upon rapamycin treatment. Single-gene defects in both of these STATs lead to early-onset autoimmunity by disrupting T-cell development and polarization. ${ }^{1,4,5,8}$ These results suggest that the TOM1 mutation affects MAPK and JAKSTAT signaling. This most likely explains the observed Th1 and Th17 cell developmental defects (Table S3) and contributes to poor regulatory T-cell function.

\section{DISCUSSION}

We associate heterozygous TOM1 mutation to a combined immunodeficiency and early-onset autoimmunity. The TOM1 mutation disrupts the TOM1-TOLLIP complex formation and the patient cells show defects in autophagy, susceptibility to apoptosis, and downregulation of multiple key signaling effectors, including ERK1/2, STAT1, and STAT5. The difference in disease severity suggest that additional genetic and environmental factors modify the phenotype. As in all ultra-rare diseases initially reported in single families, additional cases from unrelated pedigrees are needed to determine with complete certainty a causal relationship between mutations in TOM1 and the clinical phenotype described here, and to better understand the molecular pathogenesis of TOM1 mutations. ${ }^{23}$

\section{METHODS}

\section{Study participants}

The study was conducted in accordance to the principles of the Helsinki Declaration and was approved by the Helsinki University Hospital Ethics Committee. Written informed consent was obtained from the mother and father, as well as their permission for the child. Also all other participants signed written informed consent. The authors affirm that human research participants provided informed consent, for publication of the images in the Supplementary Fig. S1. 
DNA extraction and whole-exome sequencing, and validation of the candidate mutations

Genomic DNA was extracted from EDTA blood samples using Qiagen FlexiGene DNA kit (Qiagen). Libraries were processed according to Agilent SureSelect Target Enrichment System (Agilent Technologies) for Illumina Paired-End Sequencing Library (Illumina) using SureSelect Human All Exon V5 capture library (Agilent Technologies). Libraries were sequenced with $101 \mathrm{bp}$ read length (HiSeq1500 sequencing platform, Illumina), with $\sim 120 \mathrm{x}$ depth.

The read mapping, variant calling, and genome annotation were performed as described previously. ${ }^{3}$ We first analyzed the parent and child exomes separately. After variant calling, we filtered the data for coding mutations with minor allele frequency of $<0.01$ in control databases (The Exome Aggregation Consortium (ExAC), 1000 Genomes, NHLBI Exome variant server and UK TWIN and ALSPAC study cohorts (2-4), as well as in-house databases. We then focused the search on rare, damaging variants in known Mendelian disease-causing genes; however, neither patient did have damaging variants with appropriate phenotype and heritage model.

We next targeted the analysis on shared variants between the affected individuals. We filtered the data for shared coding mutations not present in control databases (The Exome Aggregation Consortium (ExAC), 1000 Genomes, NHLBI Exome variant server and UK TWIN and ALSPAC study cohorts (2-4), as well as in-house databases) (see Table S4). This analysis recovered two functionally plausible candidate variants, one in TOM1 and another in TBC1D31.

As the phenotype in the child was more severe than in the parent, we also analyzed the data for damaging, coding variants that were heterozygous in the mother, and homozygous or compound heterozygous in the parent. No plausible variants were found.

The candidate mutations were verified by sequencing from fibroblast DNA and RNA/cDNA (Eurofins Genomics Germany GmbH, Germany) (Fig. S1B). The mutant allele was expressed similarly to WT. Primers used for sequencing (GAGGAGCTGCTCATCGTCAATG), forward primer for gDNA and CDNA PCR (TOM1-forward1 CTGGAGCTCATCCCTCAGAT), reverse primer for CDNA PCR (TOM1-reverse1 TACCTCTITCCGTTGGTCAGC) and reverse primer for gDNA PCR (TOM1-reverse2 AGCTGGGATGAGAGGTTGC).

\section{B- and T-cell immunophenotyping}

Fresh EDTA blood samples or PBMCs were used for B and T lymphocyte immunophenotyping. Four or 6-color flow cytometry panel with mAbs against the surface antigens $\operatorname{lgM}, \operatorname{lgD}, \mathrm{CD} 3, \mathrm{CD} 4, \mathrm{CD} 8, \mathrm{CD} 16 / 56, \mathrm{CD} 19$, CD21, CD27, CD33, CD34, CD38, CD45, CD56, CD57, CD133, HLA-DR, CD62L, CD45RA, and CD45RO (BD Biosciences) were applied. ${ }^{24}$ The memory status of $T$ cells was studied with an antibody panel, including anti-CD45, -CD3, -CD4, -CD45RA, and -CCR7 (R\&D Systems). ${ }^{3,24}$

Evaluation of T-cell responses is described in detail elsewhere. ${ }^{24}$ For the assessment of T-cell activation, fresh mononuclear cells were stimulated for $6 \mathrm{~h}$ with anti-CD3, anti-CD28, and anti-CD49d (BD Biosciences). The cells were analyzed using a 4- or 6-color flow cytometry panel with mAbs against the antigens CD45, CD3, CD4, CD8, CD16, CD56, CD45, CD45RA, TCR- $\gamma$, CCR7, IFN- $\gamma$, and tumor necrosis factor (TNF).

\section{Analysis of T- and NK-cell cytotoxicity}

Evaluation of T- and NK-cell responses is described in detail elsewhere. ${ }^{24,25}$ For the assessment of T-cell activation and degranulation, fresh mononuclear cells (MNCs) were stimulated for $6 \mathrm{~h}$ with anti-CD3, anti-CD28, and anti-CD49d (BD Biosciences). For NK-cell degranulation, cytokine and cytotoxicity assays, fresh MNCs or FACS-sorted CD3-CD16/56 + NK cells were stimulated with $\mathrm{K} 562$ target cells for $6 \mathrm{~h}$. The cells were analyzed using 4- or 6-color flow cytometry panel with mAbs against the antigens CD45, CD3, CD4, CD8, CD16, CD56, CD45, CD45RA, TCRy, CCR7, IFN- $\gamma$ and TNF-a. Additionally, standard $4 \mathrm{~h}$ chromium $51(51 \mathrm{Cr})$-release assays were performed according to established protocols for clinical samples using magnetic bead-separated $\mathrm{CD} 3+\mathrm{CD} 8+\mathrm{T}$ cell or CD3-CD56 + NK cell subsets. ${ }^{24,25}$

\section{Phenotyping of Th17 and $\mathrm{T}_{\text {reg }}$ cells}

Phenotyping of IL-17-positive Th17 cells and $\mathrm{T}_{\text {regs }}$ is described in detail elsewhere. ${ }^{3}$ Briefly, fresh PBMCs were stimulated for $16 \mathrm{~h}$ with anti-CD3/ anti-CD28 beads in the presence of Brefeldin A. Thereafter, the cells were fixed, permeabilized and stained with anti-CD4, -CD69-APC, and IL-17A (BD
Biosciences) and analyzed with FACSAria II or FACSCanto II or LSRFortessa flow cytometer.

Th1/Th17 CD4 + memory cells were detected from whole blood by a four-color flow cytometry panel with mAbs (CD45RA-FITC, CD4-PerCP, CXCR3-APC, and CCR6-BV421 (BioLegend, San Diego, CA)) against surface antigens similarly to ref. ${ }^{26}$ eZKine ${ }^{\text {TM }}$ Th1/Th17 Whole Blood Intracellular Cytokine Kit (eBioSciences) was used to measure the INF-gamma production.

$T_{\text {regs }}$ were immunophenotyped from fresh blood with surface markers against CD4, CD25, and FOXP3 (BD Biosciences). ${ }^{3}$ For evaluation of $\mathrm{T}_{\text {reg }}$ suppressor capacity, $C D 4^{+} \mathrm{CD} 25^{+} \mathrm{CD} 127^{-} \mathrm{T}_{\text {reg }}$ cells were sorted from whole blood using Human CD4 + T Cell Enrichment Cocktail (Stemcell Technologies) and fluorescence-activated cell sorting with mAbs against CD4, CD25, and CD127 (BD Biosciences). The cells were incubated for 6 days with CFSE-labeled autologous responder T cells in a ratio of 1:2. Anti-CD3/ anti-CD28 beads (Life Technologies) were used as stimulus. CD4 ${ }^{+}$cells were analyzed using FACSAria II flow cytometer (BD Biosciences). The suppression percentage was calculated with the following formula: $100-$ [ $\left(\%\right.$ proliferation in presence of $\mathrm{T}_{\text {reg }} / \%$ proliferation in absence of $\mathrm{T}_{\text {reg }}$ ) $\mathrm{x} 100]^{3,27}$

\section{Generation of inducible Flp-In ${ }^{\mathrm{TM}}$ T-REx 293 cell lines}

The cDNA constructs containing WT and p.G307D mutant TOM1 and LRBA full length coding sequences were ordered as synthetic genes and cloned into pTO_HA_StrepllI_C_GW_FRT, ${ }^{28}$ pTO_MYC_BirA_c, ${ }^{29}$ and pCDNADEST40_3 3 V $5^{30}$ destination vectors. The TOLLIP CDNA was obtained as a gateway compatible entry-clone and cloned into PTO_HA_StrepllI_n_GW_FRT ${ }^{28}$ and pTO_MYC_BirA_n destination vectors. Generation and culture of Flp-In T-REx 293 (ThermoFischerScientific) cell lines was performed as previously described. ${ }^{28,31}$

\section{Western blotting}

For WB analysis, $5 \times 105$ Flp-In ${ }^{\mathrm{TM}}$ T-Rex 293 cells were seeded to six-well plates, induced with tetracycline, harvested to Laemmli Sample Buffer, boiled and ran to sodium dodecyl sulfate polyacrylamide gel electrophoresis (SDS-PAGE) gel. Proteins were transferred onto nitrocellulose membrane and detected with anti-HA (HA-11, Covance), anti-V5 (Invitrogen), or anti-c-Myc primary (9E10, Santa Cruz) and horseradish peroxidase (HRP)-conjugated secondary antibody. Anti-alpha Tubulin antibody (ab7291, Abcam) was used as a loading control. Signal was visualized by chemiluminescence.

\section{Immunofluorescence}

Hela cells were seeded onto coverslips on four-well plates, transiently transfected with Fugene6 (Promega) according to manufacturer's instructions. The expression of the constructs was induced with tetracycline and after $24 \mathrm{~h}$ the Hela cells were fixed with 4\% PFA. Cell were stained with anti-HA antibody, anti-V5 antibody and visualized with Alexa-488 goat antimouse IgG (Life Technologies, Thermo Fisher Scientific) or Alexa594 goat anti-rabbit IgG. Nuclei were stained with DAPI prior to mounting with Vectashield (Vector Laboratories). Fluorescent microscope, Zeiss Axio Scope.A1 (Zeiss, Oberkochen, Germany) with $\times 40$ magnification was used to image the samples. The image files were processed with Zen (Zeiss, Oberkochen, Germany), CorelDRAW X7 and ImageJ softwares.

\section{Affinity purification, BiolD (purification of BirA*), and mass spectrometry}

Affinity purification (AP) and BiolD experiments together with mass spectrometry were performed as previously described. ${ }^{29}$ Briefly, for AP $\sim 5 \times 10^{7}$ cells were lysed in HNN lysis buffer $(50 \mathrm{mM}$ HEPES pH 8.0, $150 \mathrm{mM}$ $\mathrm{NaCl}, 5 \mathrm{mM}$ EDTA, 0.5\% NP-40, $50 \mathrm{mM} \mathrm{NaF}, 1.5 \mathrm{mM}$ Na3VO4, $1.0 \mathrm{mM}$ PMSF (phenylmethanesulfonylfluoride) and $10 \mu \mathrm{l} / \mathrm{ml}$ protease inhibitor cocktail, Sigma). For BiolD $\sim 5 \times 10^{7}$ cells $(5 \times 15 \mathrm{~cm}$ dishes) in three biological replicates were induced with $2 \mu \mathrm{g} / \mathrm{ml}$ doxycycline and $50 \mu \mathrm{M}$ biotin for $24 \mathrm{~h}$. After induction, cells were washed and harvested under harsher conditions using $0.1 \%$ SDS and $80 \mathrm{U} / \mathrm{ml}$ Benzonase Nuclease (Santa Cruz Biotechnology) in HNN lysis buffer. The proteins were bound using Strep-Tactin sepharose and Bio-Spin chromatography columns (Bio-Rad) and eluted with D-biotin (Thermo Scientific). After C18-purification samples were analyzed with Orbitrap Elite ETD hybrid mass spectrometer as described in ref. ${ }^{29}$ Each sample was analyzed in triplicates. The biological 
clustering and pathway analysis was conducted using DAVID bioinformatics database. ${ }^{32}$

Small interfering RNA (siRNA) knock-down of TBC1D31

The ON-TARGETplus siRNA SMARTpool targeting human TBD1D31 (GUGAUGAUCUACAACGAAA, UGGCUGAAAUUGUUCGAUA, GCAGAUGCCU AUAGACGAA, GAUAAAUGCGGCUGUAGAA) and the non-targeting pool (UGGUUUACAUGUCGACUAA, UGGUUUACAUGUUGUGUGA, UGGUUUACA UGUUUUCUGA, UGGUUUACAUGUUUUCCUA) (Dharmacon, USA) were transfected to parental HAP1 cells (Horizon Discovery, UK) with DharmaFECT according to manufacturer's protocols using recommended $100 \mathrm{nM}$ final siRNA concentration. Twenty-four hour post transfection, the transfection medium was replaced with normal complete medium. Cells were analyzed for autophagy induction $48 \mathrm{~h}$ after transfection using FlowCellect ${ }^{\mathrm{TM}}$ Autophagy LC3 Antibody-based Assay Kit (Merck Millipore). For autophagy induction, the cells were either starved for $3 \mathrm{~h}$ (induced) or left untreated (control).

\section{Immunohistochemical staining of GRP78/BiP}

Immunohistochemisty $(\mathrm{IHC})$ of lung and duodenal biopsy paraffin sections was performed according to standard techniques using GRP78/BiP mAb (ab21685, Abcam). Biopsy slides from three healthy individuals (C1, C2, C3) were used as controls.

\section{CTLA4 staining of peripheral blood lymphocytes}

Mononuclear cells purified from heparinized blood of the two patients and two healthy controls $(C 4, C 5)$ were stimulated either with $C D 3 / C D 28$ Dynabeads (Invitrogen) and IL-2 (Proleukin), phytohemagglutinin and Ionomycin, or growth media alone, for 5 days. Cells were then evaluated for CTLA4 (Clone BNI3) expression in CD4 ${ }^{+} \mathrm{T}$ cells by flow cytometry.

\section{LC3 staining of peripheral blood mononuclear cells}

Fresh PBMCs from the patients and three healthy controls $(C 6, C 7, C 8)$ were incubated overnight in RPMI supplemented with 10\% FCS and $1 \%$ Penicillin-Streptomycin. To analyze the LC3 expression by FACS, the next day cells were either serum-starved or let grow for $6 \mathrm{~h}$. The LC3 staining was done according to FlowCellect ${ }^{\mathrm{TM}}$ Autophagy LC3 Antibody-based Assay Kit (Merck Millipore). The LC3 expression was measured according to kit instructions using Guava easyCyte 6-2 L (Merck Millipore). All samples were analyzed in triplicate.

\section{Apoptosis assay}

Fresh PBMCs from the patients and three healthy controls $(C 6, C 7, C 8)$ were stained according to ViaCount ${ }^{\circledR}$ Reagent (Merck Millipore) instructions immediately after isolation. The amount of viable, apoptotic and dead cells was measured according to instructions using Guava easyCyte 6-2 L (Merck Millipore).

\section{Phospho-flow analysis by mass cytometry}

We performed skin biopsy to establish fibroblast cell lines from both patients and a healthy control (C9). Once established, the cells were incubated for $16 \mathrm{~h}$ with $500 \mathrm{nM}$ rapamycin or left untreated. The following day cells were fixed with paraformaldehyde, permeabilized with cold methanol, and stained with Maxpar Signaling I Panel Kit according to Maxpar Phoshophoprotein Staining Protocol provided by manufacturer (Fluidigm). Data was collected on a CyTOF mass cytometer (Fluidigm). For data analysis we utilized Cytobank premium (cytobank.org).

\section{Reporting summary}

Further information on experimental design is available in the Nature Research Reporting Summary linked to this paper.

\section{DATA AVAILABILITY}

The raw data that support the findings of this study are available from the corresponding author upon reasonable request. The sequencing data from the two study patients are available through the Institute for Molecular Medicine Finland (FIMM) Data Access Committee (DAC) for authorized researchers who have IRB/ethics approval and an institutionally approved study plan. For more details, please contact the FIMM DAC (http://fimm-dac@helsinki.fi).

\section{ACKNOWLEDGEMENTS}

We sincerely thank the family for their co-operation and patience. Personnel at Institute for Molecular Medicine Finland (FIMM) Science for Life laboratory Stockholm are acknowledged for their expert technical assistance. Sini Miettinen is thanked for excellent technical assistance. Eira Leinonen and Auli Saarinen are thanked for their skillful assistance throughout the project. The Academy of Finland, Finnish Medical Foundation, Sigrid Juselius Foundation, Karolinska Institutet Research Foundation, Swedish Research Council, and Strategic Research Program in Diabetes supported this work.

\section{AUTHOR CONTRIBUTIONS}

E.H, S.K., and M.V. designed the study, performed experiments, and wrote the manuscript. E.H and S.K. contributed equally, and are co-first authors. V.G., S.C.C., S.M., H.R., and Y.B. performed FACS analyses and blood immunophenotyping. X.L. performed cell biology and microscopy. P.K. and J.L. performed immunohistochemistry and reviewed the histopathology. V.L., M.S., and K.H. provided clinical care for the patients. All authors read and approved the manuscript.

\section{ADDITIONAL INFORMATION}

Supplementary Information accompanies the paper on the npj Genomic Medicine website (https://doi.org/10.1038/s41525-019-0088-5).

Competing interests: M.S. has received honoraria from CSL Behring and Octapharma. The other authors declare no competing interests.

Publisher's note: Springer Nature remains neutral with regard to jurisdictional claims in published maps and institutional affiliations.

\section{REFERENCES}

1. Andersson, E. I. et al. High incidence of activating STAT5B mutations in CD4-positive T-cell large granular lymphocyte leukemia. Blood 128, 2465-2468 (2016).

2. Grimbacher, B., Warnatz, K., Yong, P. F. K., Korganow, A. S. \& Peter, H. H. The crossroads of autoimmunity and immunodeficiency: Lessons from polygenic traits and monogenic defects. J. Allergy Clin. Immunol. 137, 3-17 (2016).

3. Haapaniemi, E. M. et al. Autoimmunity, hypogammaglobulinemia, lymphoproliferation, and mycobacterial disease in patients with activating mutations in STAT3. Blood 125, 639-648 (2015).

4. Kanai, T., Jenks, J. \& Nadeau, K. C. The STAT5b pathway defect and autoimmunity. Front. Immunol. 3, 234 (2012).

5. Klammt, J. et al. Dominant-negative STAT5B mutations cause growth hormone insensitivity with short stature and mild immune dysregulation. Nat. Commun. 9, 2105 (2018).

6. Noack, M. \& Miossec, P. Th17 and regulatory T cell balance in autoimmune and inflammatory diseases. Autoimmun. Rev. 13, 668-677 (2014).

7. Pfajfer, L. et al. Mutations affecting the actin regulator WD repeat-containing protein 1 lead to aberrant lymphoid immunity. J. Allergy Clin. Immunol. https:// doi.org/10.1016/j.jaci.2018.04.023 (2018).

8. Toubiana, J. et al. Heterozygous STAT1 gain-of-function mutations underlie an unexpectedly broad clinical phenotype. Blood 127, 3154-3164 (2016).

9. Lo, B. et al. Autoimmune disease. Patients with LRBA deficiency show CTLA4 loss and immune dysregulation responsive to abatacept therapy. Science 349, 436-440 (2015).

10. Lohr, N. J. et al. Human ITCH E3 ubiquitin ligase deficiency causes syndromic multisystem autoimmune disease. Am. J. Hum. Genet. 86, 447-453 (2010).

11. Watkin, L. B. et al. COPA mutations impair ER-Golgi transport and cause hereditary autoimmune-mediated lung disease and arthritis. Nat. Genet. 47, 654-660 (2015).

12. Tumbarello, D. A. et al. Autophagy receptors link myosin VI to autophagosomes to mediate Tom1-dependent autophagosome maturation and fusion with the lysosome. Nat. Cell Biol. 14, 1024-1035 (2012).

13. Katoh, Y. et al. Tollip and Tom 1 form a complex and recruit ubiquitin-conjugated proteins onto early endosomes. J. Biol. Chem. 279, 24435-24443 (2004).

14. Xiao, S. et al. Tom 1 modulates binding of tollip to phosphatidylinositol 3phosphate via a coupled folding and binding mechanism. Structure 23, 1910-1920 (2015). 
15. Taipale, M. Disruption of protein function by pathogenic mutations: common and uncommon mechanisms (1). Biochem. Cell Biol. 97, 46-57 (2019).

16. Kowalski, E. J. A. \& Li, L. Toll-interacting protein in resolving and non-resolving. Inflamm. Front. Immunol. 8, 511 (2017).

17. Roux, K. J., Kim, D. I., Raida, M. \& Burke, B. A promiscuous biotin ligase fusion protein identifies proximal and interacting proteins in mammalian cells. J. Cell Biol. 196, 801-810 (2012).

18. Lopez-Herrera, G. et al. Deleterious mutations in LRBA are associated with a syndrome of immune deficiency and autoimmunity. Am. J. Hum. Genet. 90, 986-1001 (2012).

19. Marino, G., Niso-Santano, M., Baehrecke, E. H. \& Kroemer, G. Self-consumption: the interplay of autophagy and apoptosis. Nat. Rev. Mol. Cell Biol. 15, 81-94 (2014).

20. Levine, B. \& Kroemer, G. Autophagy in the pathogenesis of disease. Cell 132, 27-42 (2008).

21. Deegan, S., Saveljeva, S., Gorman, A. M. \& Samali, A. Stress-induced self-cannibalism: on the regulation of autophagy by endoplasmic reticulum stress. Cell Mol. Life Sci. 70, 2425-2441 (2013).

22. Kuehn, H. S. et al. Immune dysregulation in human subjects with heterozygous germline mutations in CTLA4. Science 345, 1623-1627 (2014).

23. Fresard, L. \& Montgomery, S. B. Diagnosing rare diseases after the exome. Cold Spring Harb. Mol. Case Stud. 4. https://doi.org/10.1101/mcs.a003392 (2018).

24. llander, M. et al. Enlarged memory T-cell pool and enhanced Th1-type responses in chronic myeloid leukemia patients who have successfully discontinued IFNalpha monotherapy. PLOS ONE 9, e87794 (2014).

25. Chiang, S. C. et al. Comparison of primary human cytotoxic T-cell and natural killer cell responses reveal similar molecular requirements for lytic granule exocytosis but differences in cytokine production. Blood 121, 1345-1356 (2013).

26. Ma, C. S. et al. Monogenic mutations differentially affect the quantity and quality of $\mathrm{T}$ follicular helper cells in patients with human primary immunodeficiencies. $J$. Allergy Clin. Immunol. 136, 993-1006 (2015).
27. Ruitenberg, J. J., Boyce, C., Hingorani, R., Putnam, A. \& Ghanekar, S. A. Rapid assessment of in vitro expanded human regulatory $\mathrm{T}$ cell function. J. Immunol. Methods 372, 95-106 (2011).

28. Varjosalo, M. et al. The protein interaction landscape of the human CMGC kinase group. Cell Rep. 3, 1306-1320 (2013).

29. Heikkinen, T. et al. Somatic MED12 nonsense mutation escapes mRNA decay and reveals a motif required for nuclear entry. Hum. Mutat. 38, 269-274 (2017).

30. Varjosalo, M. et al. Application of active and kinase-deficient kinome collection for identification of kinases regulating hedgehog signaling. Cell 133, 537-548 (2008).

31. Karpanen, T. et al. Functional interaction of VEGF-C and VEGF-D with neuropilin receptors. FASEB J. 20, 1462-1472 (2006).

32. Huang da, W., Sherman, B. T. \& Lempicki, R. A. Systematic and integrative analysis of large gene lists using DAVID bioinformatics resources. Nat. Protoc. 4, 44-57 (2009).

Open Access This article is licensed under a Creative Commons Attribution 4.0 International License, which permits use, sharing adaptation, distribution and reproduction in any medium or format, as long as you give appropriate credit to the original author(s) and the source, provide a link to the Creative Commons license, and indicate if changes were made. The images or other third party material in this article are included in the article's Creative Commons license, unless indicated otherwise in a credit line to the material. If material is not included in the article's Creative Commons license and your intended use is not permitted by statutory regulation or exceeds the permitted use, you will need to obtain permission directly from the copyright holder. To view a copy of this license, visit http://creativecommons. org/licenses/by/4.0/.

(c) The Author(s) 2019 